\title{
GMR
}

\section{Comparative transcriptome analysis of testes and ovaries for the discovery of novel genes from Amur sturgeon (Acipenser schrenckii)}

\author{
S.B. Jin ${ }^{2 *}$, Y. Zhang ${ }^{1 *}$, X.L. Dong ${ }^{1}$, Q.K. Xi ${ }^{2}$, D. Song ${ }^{2}$, H.T. Fü and D.J. Sun ${ }^{1}$ \\ ${ }^{1}$ Heilongjiang River Fisheries Research Institute, \\ Chinese Academy of Fishery Sciences, Haebin, China \\ ${ }^{2}$ Key Laboratory of Freshwater Fisheries and Germplasm Resources Utilization, \\ Ministry of Agriculture, Freshwater Fisheries Research Center, \\ Chinese Academy of Fishery Sciences, Wuxi, China \\ ${ }^{*}$ These authors contributed equally to this study. \\ Corresponding author: D.J. Sun \\ E-mail:13845059350@163.com \\ Genet. Mol. Res. 14 (4): 18913-18927 (2015) \\ Received August 16, 2015 \\ Accepted October 30, 2015 \\ Published December 28, 2015 \\ DOI http://dx.doi.org/10.4238/2015.December.28.40
}

\begin{abstract}
Sturgeons (Acipenser schrenckii) are of high evolutionary, economic, and conservation value, and caviar isone of the most valuable animal food products in the world. The Illumina HiSeq2000 sequencing platform was used to construct testicular and ovarian transcriptomes to identify genes involved in reproduction and sex determination in $A$. schrenckii. A total of 122,381 and 114,527 unigenes were obtained in the testicular and ovarian transcriptomes, respectively, with average lengths of 748 and $697 \mathrm{bp}$. A total of 46,179 genes were matched to the nonredundant nr database. GO $(31,266)$, KEGG $(39,712)$, and COG analyses $(20,126)$ were performed to identify potential genes and their functions. Twenty-six gene families involved in reproduction and sex determination were identified from the $A$. schrenckii testicular and ovarian transcriptomes based on functional annotation of non-redundant transcripts and comparisons with the published literature. Furthermore, 1309 unigenes
\end{abstract}


showed significant differences between the testes and ovaries, including 782 genes that were up-regulated in the testes and 527 that were upregulated in the ovaries. Eleven genes were involved in reproduction and sex determination mechanisms. Furthermore, 19,065 simple sequence repeats (SSRs) were identified in the expressed sequence tagged dataset, and 190,863 and 193,258 single nucleotide polymorphisms (SNPs) were obtained from the testicular and ovarian transcriptomic databases, respectively. This study provides new sequence information about $A$. schrenckii, which will provide a basis for the further study of reproduction and sex determination mechanisms in Acipenser species. The potential SSR and SNP markers isolated from the transcriptome may shed light on the evolution and molecular ecology of Acipenser species.

Key words: Acipenser schrenckii; Testes; Ovaries; Transcriptome; Reproduction

\section{INTRODUCTION}

Amur sturgeon (Acipenser schrenckii), also known as a living fossil, is one of the most primitive Actinopterygii species. Amur sturgeon is a species of fish belonging to the Acipenseridae family that can be found in the Amur River, Songhua River, and Heilong River (Li et al., 2012). Sturgeon species have become very popular in Chinese aquaculture for their considerable value in the economy and in ecology. Sturgeons accounted for $15 \%$ of the total sturgeon production in Chinese aquaculture (Wei et al., 2011). In recent years, sturgeons have received a considerable amount of interest in many countries (Wang et al., 2009a). However, in the last few decades, increasingly intense fishing practices have caused a dramatic decline in the distribution and population of wild stocks. Most sturgeon species are at risk of extinction; therefore, the International Union for Conservation of Nature has listed sturgeons as one of the most critically endangered group of species. In addition, the high economic value of these animals is a further advantage for the transcriptome characterization of sturgeon species. Sturgeon eggs of are sold as caviar and represent one of the most highly prized foods of animal origin (Ludwig, 2008). Female sturgeons, which can produce an overwhelming number of eggs, are desirable in the sturgeon aquaculture program. However, anoverridingproblem for aquaculture caviar producers is that $50 \%$ of the animals are profitless males, which need to be discarded from production as quickly as possible in order to minimize expenditure and maximize space. Thus, in order to maintain their sustainable development, understanding the reproduction and sex-differentiation mechanism of $A$. schrenckii is an urgent topic of research.

The transcriptome includes all the transcripts, mRNA, and non-coding RNA in one cell or in a population of cells during a specific developmental stage or in response to a particular physiological condition, and is determined using high-throughput technology (Mardis, 2008). Transcriptome analysis provides a foundation for gene structure and function research, and reveals the timing ofgene expression and associated regulation mechanisms. The development of nextgeneration sequencing (NGS) technologies allows the acquisition of more sequence data per run at a substantially lower cost than are obtained using long-read technologies (Huang and Marth, 2008). Compared with microarray methods, NGS, developed by Illumina/Solexa, is able to generate over 
one billion bases of high quality sequences per run at less than $1 \%$ of the cost of capillary-based methods, and is expected to dominate the future analysis of eukaryotic transcriptomes (Cloonan and Grimmond, 2008; Morozova and Marra, 2008; Wang et al., 2009b). Transcriptome analysis is now being widely applied to elucidate genetic factors that confer economically significant traits and/or phenotypes to the management of genetic diversity in cultured fish species. To date, only a limited number of unique sequences have been obtained for $A$. schrenckii and Acipenser species. In this study, we investigated the gene expression profile in testes and ovariesof $A$. schrenckii.

We aimed first to generate the first $A$. schrenckii comparative transcriptome analysis using cDNA reverse-transcribed from mRNA isolated from the testes and ovaries, in order to identify candidate genes that are involved in reproduction and that differ in their expression between males and females. Our second goal was to obtaina global view of the molecular mechanism(s) that underlie howgene expression influences reproduction and gonad development in $A$. schrenckii.

\section{MATERIAL AND METHODS}

\section{Experimental fishes}

Amur sturgeons were collected from the Engineering and Technology Center of Sturgeon Breeding and Cultivation of Chinese Academy of Fishery Science in Beijing, China. Eight healthy $A$. schrenckii (four males and four females) with a wet weight ranging from 12.7 to $28.5 \mathrm{~kg}$ (average = $22.36 \mathrm{~kg}$ ), and a total length ranging from 130.2 to $164.7 \mathrm{~cm}$ (average $=158.67 \mathrm{~cm}$ ) were obtained for transcriptome construction. In order to prevent total RNA degradation, the testes and ovaries were collected and immediately frozen in liquid nitrogen until RNA was extracted for transcriptome sequencing.

\section{RNA isolation for RNA-seq}

The prepared testes and ovaries were pooled to provide sufficient RNA for transcriptome sequencing. Total RNA was extracted using the UNIQ-10 Column Trizol Total RNA Isolation Kit (Sangon) following the manufacturer protocol. To confirm the purity of the RNA sample, the OD260/280 and OD260/230 were measured, and ranged from 1.8 to 2.0 and $>2.0$, respectively. The total concentration of the RNA sample was adjusted to $>5 \mathrm{mg}$ to guarantee transcriptome quality. RNA integrity was confirmed using 2100 Bioanalyzer (Agilent Technologies, Inc.) with a minimum RNA integrity number (RIN) value of 7.0 and an ABI StepOnePlus Real-Time PCR System. The samples used for transcriptome analysis were prepared using a TruseqTM RNA Sample Prep Kit (Illumina) according to the manufacturer recommendation. Briefly, mRNA was isolated from total RNA using oligo (dT) magnetic beads. mRNA was cut into short fragments by the addition of fragmentation buffer in a Thermomixer at a suitable temperature. First-strand cDNA was synthesized using random hexamer-primers, using these short fragments as templates. RNaseH, buffer, dNTPs, and DNA polymerase I were used to synthesize second-strand cDNA. Short fragments were purified with aPCR extraction kit (Takara Bio, Inc.). Sequencing adapters were ligated to form short fragments and then resolved by agarose gel electrophoresis. Fragments were selected and purified, and subsequently PCR amplified to create the final cDNA library template. 


\section{Analysis of the transcriptome results}

The transcriptome was sequenced using the Illumina HiSeq ${ }^{\mathrm{TM}} 2000$ and each sample was sequenced twice. Four fluorescently labeled nucleotides and a specialized polymerase were used to determine the clusters, base-by-base in parallel. The size of the library was approximately 200 bp and both ends of the library were sequenced. The 200-bp raw paired-end reads were generated on the Illumina sequencing platform. Image deconvolution and quality value calculations were performed using Illumina GA pipeline v1.6. The raw reads were cleaned by removing adaptor sequences, empty reads, and low quality sequences (reads with unknown sequences ' $N$ ' or less than25 bp). The clean reads were assembled into non-redundant transcripts using the Trinity, which has been developed specifically for the de novo assembly of transcriptomes using short reads. To obtain non-redundant transcripts, we removed short sequences (shorter than $100 \mathrm{bp}$ in length) and partially overlapping sequences. The resulting sequences were used for BLAST searches and annotation against the $\mathrm{Nr}$ protein, the SwissProt, COG, and KEGG databases using an E-value cut-off of 1025. Functional annotation by GO terms (www.geneontology.org) was analyzed by the Blast2go software. The COG and KEGG pathway annotations were performed using Blast all software against the COG and KEGG databases.

\section{Expression analysis}

The abundance of each tag was normalized to one transcriptper million, in order to allow comparison between testes and ovaries. The low quality sequences, including ambiguous nucleotides and adaptor sequences, were removed, in order to obtain cleaned raw reads. Calculation of unigene expression was conducted using RSEM software, which is an accurate and user-friendly software tool for quantifying transcript abundances from RNASeq data (Li, 2011). It is particularly useful for quantification with de novo transcriptome assemblies because it does not rely on the existence of a reference genome (Li, 2011).

\section{RESULTS}

\section{Construction and characterization of the normalized cDNA library}

The testicular and ovarian transcriptomes yielded approximately 60.8 million and 55.9 million 200-bp paired-end raw reads, respectively. After eliminating adapter sequences and filtering out low-quality reads ( $<25 \mathrm{bp}$ in each read) before de novo assembly by the SeqPreq program, the testicular and ovarian transcriptomes yielded 6,051,265,200 and 5,592,689,200 highquality transcriptome reads, respectively. The Q20 percentages and GC content of the testicular transcriptomes were 98.25 and $48.32 \%$, respectively, whereas those of the ovarian transcriptome were 98.10 and $49.24 \%$, respectively.

A. schrenckii is a non-model organism, for which there is no reference genome sequence. The raw data were therefore assembled de novo using the Trinity program. Totals of 278,257 and 255,179 contigs were assembled from the short, clean reads of the testicular and ovarian transcriptomes, respectively, with median lengths of 292 and $297 \mathrm{bp}$. From these contigs, 122,381 and 114,527 unigenes were obtained from the testicular and ovarian transcriptomes, respectively, with mean lengths of 748 and 697 bp because of alternative splicing; thus, two or more contigs 
may be matched to one unigene (Table 1). Most of the unigenes were 1-200 bp (27.09\% for the testicular transcriptome and $27.55 \%$ for the ovarian transcriptome) and 201-400 bp (27.48 for the testicular transcriptome and $27.81 \%$ for the ovarian transcriptome) in length, followed by $401-600$ bp $(11.11 \%$ for the testes and $11.26 \%$ for the ovaries) and $601-800 \mathrm{bp}(7.10 \%$ for the testicular transcriptome and $7.00 \%$ for the ovarian transcriptome) (Figure 1).

Table 1. Summary of Illumina HiSeq 2000 assembly and analysis of Acipenser schrenckii transcriptomic sequences.

\begin{tabular}{lrr}
\hline & \multicolumn{1}{c}{ Testes } & Ovaries \\
\hline Total unigenes & 122,381 & 114,527 \\
Total contigs & 278,257 & 255,179 \\
Total residues & $60,512,652$ & $55,926,892$ \\
Average length & 748 & 697 \\
Largest unigenes & 8,126 & 8,317 \\
Smallest unigenes & 141 & 129 \\
\hline
\end{tabular}

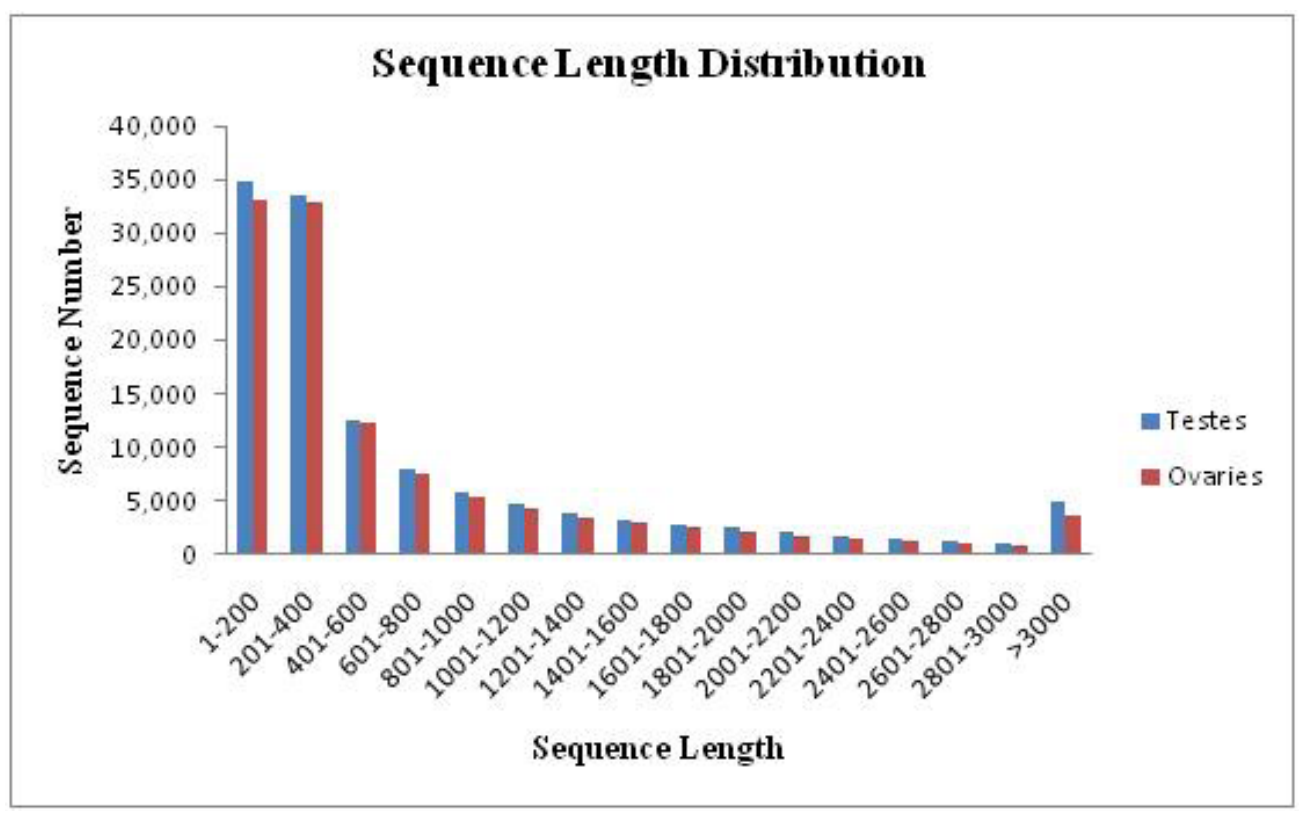

Figure 1. Contig length distribution of Acipenser schrenckii transcriptomic expressed sequence tags (ESTs).

\section{Annotation analysis}

To identify their putative functions, all unigenes were compared with the non-redundant protein database and nucleotide sequences in NCBI using Blastp and Blastx at an E-value of $<10^{-5}$ in the priority order of the Kyoto Encyclopedia of Genes and Genomes (KEGG) database, Gene Ontology (GO), and Cluster of Orthologous Groups (COG) database.

GO terms were assigned to 31,266 A. schrenckii contigs based on BLAST matches to proteins with known functions, including 45,126 sequences that were assigned to the molecular 
function category, 132,172 to the cellular component category, and 203,572 to the biological process component. The matched contigs comprised 60 functional groups (Figure 2). The number of contigs in each GO term ranged from 1 to 25,099. "Cellular process", "Cell part", "Binding", and "Single-organism process" had more than 20,000 abundant contigs, whereas there were also 12 functional groups in which the numbers of abundant contigs were less than 100 .

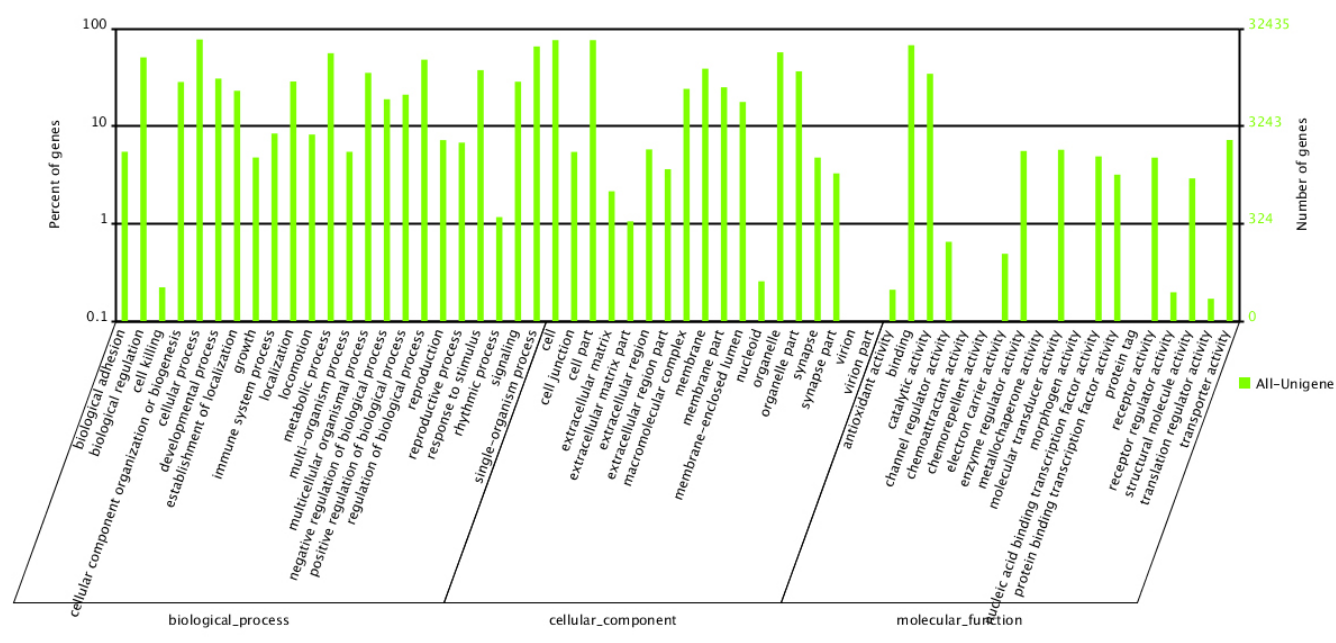

Figure 2. Gene ontology classification of non-redundant transcripts.

In addition, 20,126 unigenes matched known genes in the COG database. Based on their predicted functions, these unigenes were classified into 25 functional categories. Among these 25 functional categories, the cluster for "General function prediction only" represented the largest group with 7,913 unique sequences, followed by "Replication, recombination, and repair" (4123), "Translation, ribosomal structure, and biogenesis" (3513), "Cell cycle control, cell division, chromosome partitioning" (3145), and "Transcription" (3453). "Clusters for RNA processing and modification", "Intracellular trafficking", "Secretion vesicular transport", "Extracellular structure and nuclear structure" represented the smallest of groups, in which the numbers of sequences were $<200$ (Figure 3).

The KEGG database can map unique sequence to defined metabolic pathways, in order to identify the role or functions of potential candidate transcripts in biological pathways in the ladybird. A total of 39,712 unigenes matched to the metabolic pathways in the KEGG Pathway database, accounting for 258 predicted metabolic pathways, and were grouped into "Amino acid metabolism", "Genetic information processing", "Cellular processes", and "Environmental information processing". The numbers of unique sequences in various pathways ranged from 1 to 3613 . The main metabolic pathways of unique sequences in the $A$. schrenckii transcriptome were "Regulation of actin cytoskeleton", "Focal adhesion", "Pathways in cancer", "Tight junction", "Endocytosis", and "MAPK signaling pathway", in which the numbers of unique sequences exceeded 1200.

\section{Reproduction- and development related genes}

Twenty-six reproductive gene families were identified from the testicular and ovarian 
transcriptomes constructed from $A$. schrenckii, based on the functional annotation of non-redundant transcripts (Table 2). Most of these functional genes were identified through comparisons with published data for other species (Chen et al., 2006; Hale et al., 2010; Vidotto et al., 2013). However, some were identified based on their GO classification and COG cluster. Double-sex and Mab-3related transcription factor 1 (DMRT1), forkhead box L2 (FOXL2), Sex determining region of $Y$ chromosome-related high mobility group-box gene 9 (SOX9), and Feminization-1 (FEM1) were screened from the $A$. schrenckii transcriptome database, and were identified as playing essential roles in reproduction and development mechanisms in vertebrates and mammals.

Table 2. Reproduction-related ESTs identified in the Acipenser schrenckii transcriptome.

\begin{tabular}{|c|c|c|c|c|}
\hline Transcripts & Length (bp) & E-value & Accession No. & Hits \\
\hline Dmrt1 & $248-1274$ & 1.00E-06 & gi|23428499|gb|AY057061.1| & 7 \\
\hline Foxl2 & $254-2820$ & 0 & sp|Q6VFT6|FOXL2_PIG & 5 \\
\hline sox & $200-1806$ & 0 & gi|58465464|gb|AAW78521.1| & 112 \\
\hline SRY & 236-4111 & $1.00 \mathrm{E}-08$ & gi|170595123|ref|XP_001902255.1| & 30 \\
\hline GnRH & $580-1146$ & $3.00 \mathrm{E}-69$ & gi|465967294|gb|EMP31298.1| & 9 \\
\hline Estrogen receptor & $698-1679$ & 0 & gi|291190839|ref|NP_001167409.1| & 17 \\
\hline FHL3 & 1244 & $1.00 \mathrm{E}-162$ & gi|125840523|ref|XP_695478.2| & 1 \\
\hline FEM1 & $938-3510$ & 1.00E-79 & gi|348501380|ref|XP_003438248.1| & 5 \\
\hline ATRX & 229-4615 & $1.00 \mathrm{E}-10$ & gi|126631742|gb|AAI34027.1| & 13 \\
\hline Androgen receptor & $1562-2678$ & $1.00 \mathrm{E}-80$ & gi|224067351|ref|XP_002193622.1| & 11 \\
\hline Wnt & $541-1986$ & 0 & gi|147903413|ref|NP_001083829.1| & 58 \\
\hline FGFR & $1750-3386$ & $1.00 \mathrm{E}-47$ & gi|190888207|gb|ACE95859.1| & 29 \\
\hline Chitinase & 273-2059 & $1.00 \mathrm{E}-33$ & gi|327271191|ref|XP_003220371.1| & 11 \\
\hline Cathepsin & $221-1715$ & 0 & gi|432870116|ref|XP_004071815.1| & 53 \\
\hline Cytochrome & $441-4113$ & 0 & gi|111115685|gb|DQ7786406.1| & 132 \\
\hline Kazal-type serine proteinase inhibitor & 393 & $2.00 \mathrm{E}-09$ & gi|58081895|emb|CAI46283.1| & 1 \\
\hline Spermatogenesis-associated protein & $531-2573$ & 0 & gi|218931210|ref|NP_001073660.2| & 27 \\
\hline Carboxylesterase & $1182-3587$ & $1.00 \mathrm{E}-54$ & sp|Q99K10|NLGN1_M̄MOUSE & 42 \\
\hline Peritrophin & 902 & $5.00 \mathrm{E}-06$ & gi|307180562|gb|EFN68518.1| & 1 \\
\hline Heat Shock Protein & $589-2461$ & 0 & gi|432964405|ref|XM_004086882.1| & 86 \\
\hline Gametocyte-specific factor & 755 & $1.00 \mathrm{E}-53$ & gi|225714933|gb|ВT078889.1| & 1 \\
\hline VASA-like protein & $731-779$ & $4.00 \mathrm{E}-08$ & gi|270610556|gb|ACZ92304.1| & 2 \\
\hline Vitellogenin & $200-616$ & $1.00 \mathrm{E}-08$ & gi|305690594|gb|ADM64616.1| & 6 \\
\hline Vitellogenin receptor & 1236 & $1.00 \mathrm{E}-14$ & gi|185135214|ref|NP_001117847.1| & 1 \\
\hline Kunitz-type protease inhibitor 1 & $246-4825$ & $2.00 \mathrm{E}-07$ & gi|115527831|gb|AAI24740.1| & 17 \\
\hline Oocyte zinc finger protein & $213-1134$ & $1.00 \mathrm{E}-21$ & gi|326680600|ref|XP_003201569.1| & 5 \\
\hline
\end{tabular}

Genes differentially expressed between the testes and ovaries were investigated. A total of 1309 unigenes showed significant differences between the testicular and ovarian transcriptomes, including 782 up-regulated unigenes in the testes and 527 in the ovaries. With a view to applicability in caviar production, these 527 genes in the ovary, which may promote ovarian development, were of interest in this study (Figure 4). Among these significantly differentially expressed genes, 11 reproductive and developmental genes were identified (Table 3).

\section{Identification of SNP and SSR markers}

A large number of simple sequence repeat (SSR) and single nucleotide polymorphism (SNP) markers were obtained. A total of 19,542 SSRs were obtained in the transcriptomic dataset, including 53.02\% di-nucleotide, 35.62\% trinucleotide, and $11.36 \%$ tetra/penta/hexanucleotide repeats (Figure 5). Among the di-nucleotide repeat motifs, (GT/TG)n, (GA/AG)n, and (CA/AC)n 
were the three dominant di-nucleotide types $(N>2000)$. Among the tri-nucleotide repeat motifs, (GGA/GAG/AGG)n had a frequency of $18.01 \%$ and was the most common type, followed by (GCA/ CAG/AGC)n, (GCT/CTG/TGC)n, and (ATA/TAA/AAT)n ( $>650)$. There was a bias towards trinucleotide repeat motifs composed of $G$ and $A$.

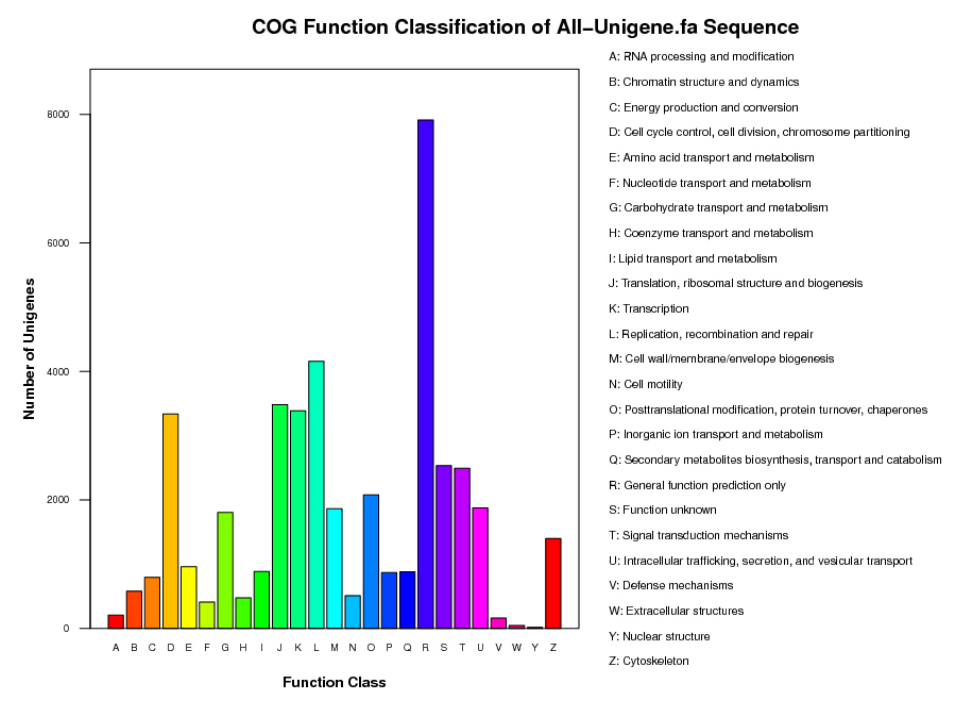

Figure 3. Cluster of orthologous groups (COG) classification of putative proteins.

\begin{tabular}{|c|c|c|c|c|}
\hline \multirow[t]{2}{*}{ Unigene name of top BLASPX hit } & \multirow[t]{2}{*}{ Length (bp) } & \multirow[t]{2}{*}{ E-value } & \multicolumn{2}{|c|}{ Expression level } \\
\hline & & & Testes & Ovaries \\
\hline Growth hormone & 977 & $1.00 \mathrm{E}-146$ & 4223 & 14590 \\
\hline SRY-related box 10 & 1271 & $3.00 \mathrm{E}-46$ & 77 & 151 \\
\hline Cathepsin F-like & 1669 & 0 & 299 & 744 \\
\hline Cytochrome P450 & 658 & $9.00 \mathrm{E}-61$ & 26 & 3 \\
\hline Cytochrome P4501B & 2665 & 0 & 9 & 110 \\
\hline Heat shock protein 10 & 765 & $2.00 \mathrm{E}-42$ & 183 & 67 \\
\hline Vasa-like protein & 779 & $4.00 \mathrm{E}-08$ & 59 & 118 \\
\hline Serine protease HTRA1B precursor & 1822 & 0 & 36 & 78 \\
\hline KRAB domain-containing zinc finger protein & 3533 & $2.00 \mathrm{E}-29$ & 23 & 59 \\
\hline Spermatogenesis & 5042 & 0 & 36 & 74 \\
\hline Carboxylesterase activity & 2456 & $9.00 \mathrm{E}-116$ & 311 & 121 \\
\hline
\end{tabular}

SNPs were identified from alignments of multiple sequences used for contig assembly. By excluding those with a mutation frequency of bases less than $1 \%$, a total of 190,863 and 193,258 SNPs, respectively, were obtained from the testicular and ovarian transcriptomic databases (Figure 6). In the testicular transcriptome, 113,226 were putative transitions (Ts) and 77,637 were putative transversions (Tv), giving a mean Ts: Tv ratio of 1.46:1, while in the ovarian transcriptome, 114,304 were putative transitions (Ts) and 78,954 were putative transversions (Tv), giving a mean Ts: Tv ratio of 1.45:1. The AG/GA and CT/TC SNPs were the most common in both transcriptomes, whereas CA/AC, AT/TA, CG/GC, and TG/GT types were the most infrequent SNP types, because of differences in the base structure and the number of hydrogen bonds between different bases. 


\section{Expression Level C1 vs C2}

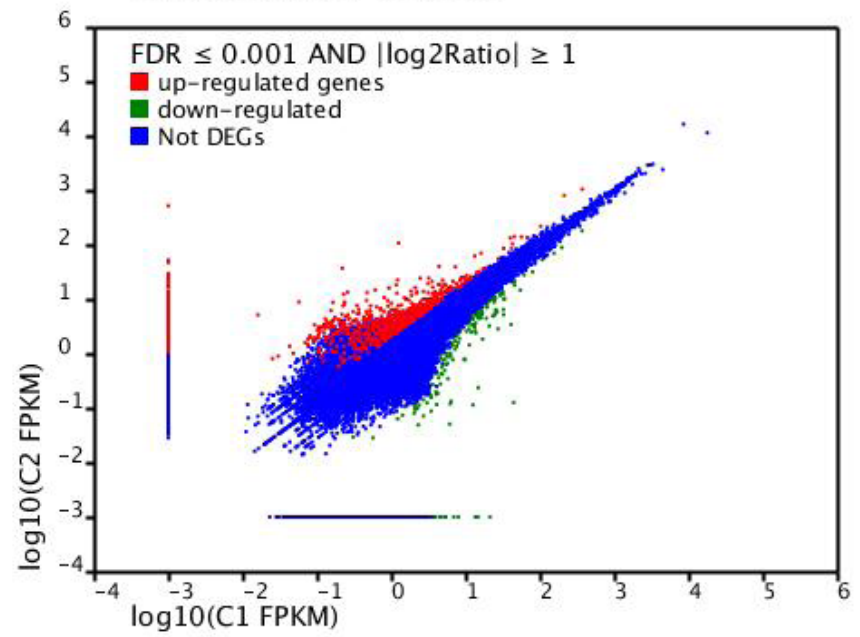

Figure 4. Differentially expressed gene (DEG) analysis of unigenes in the testes and ovaries of Acipenser schrenckii.

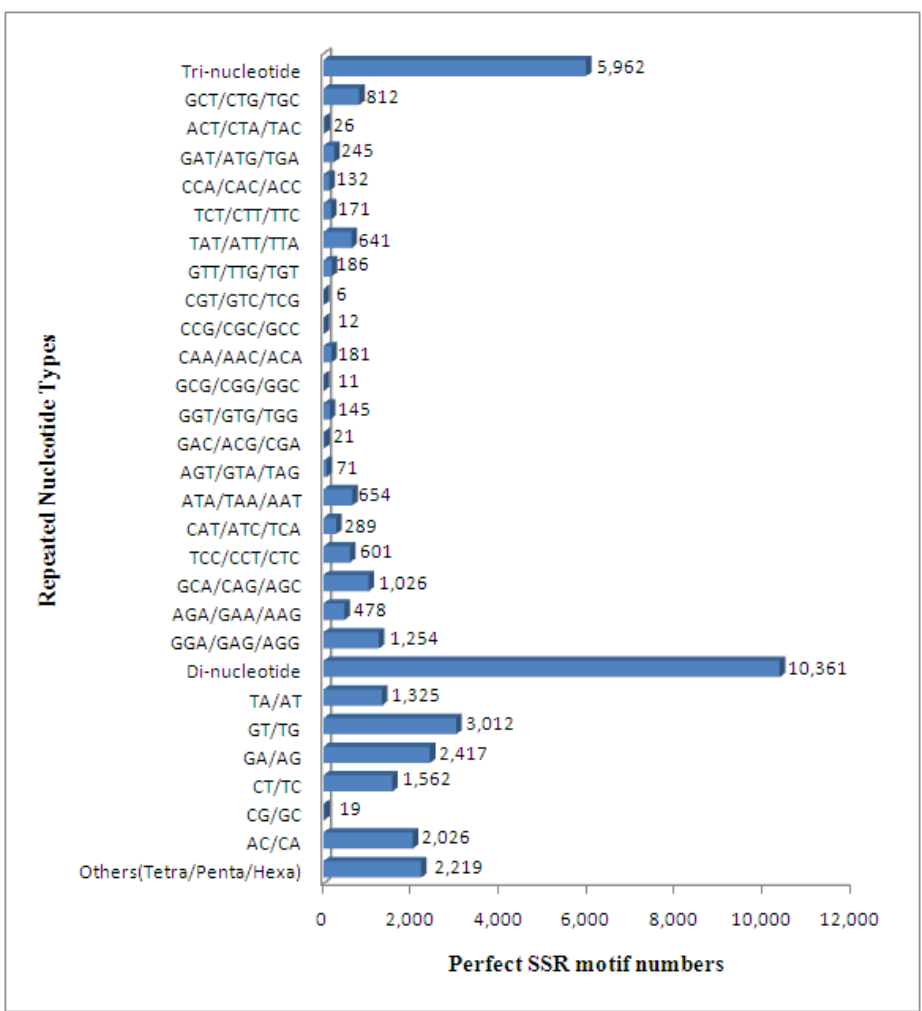

Figure 5. Distribution of simple sequence repeat (SSR) nucleotide classes among different nucleotide types found in the transcriptome of Acipenser schrenckii. 


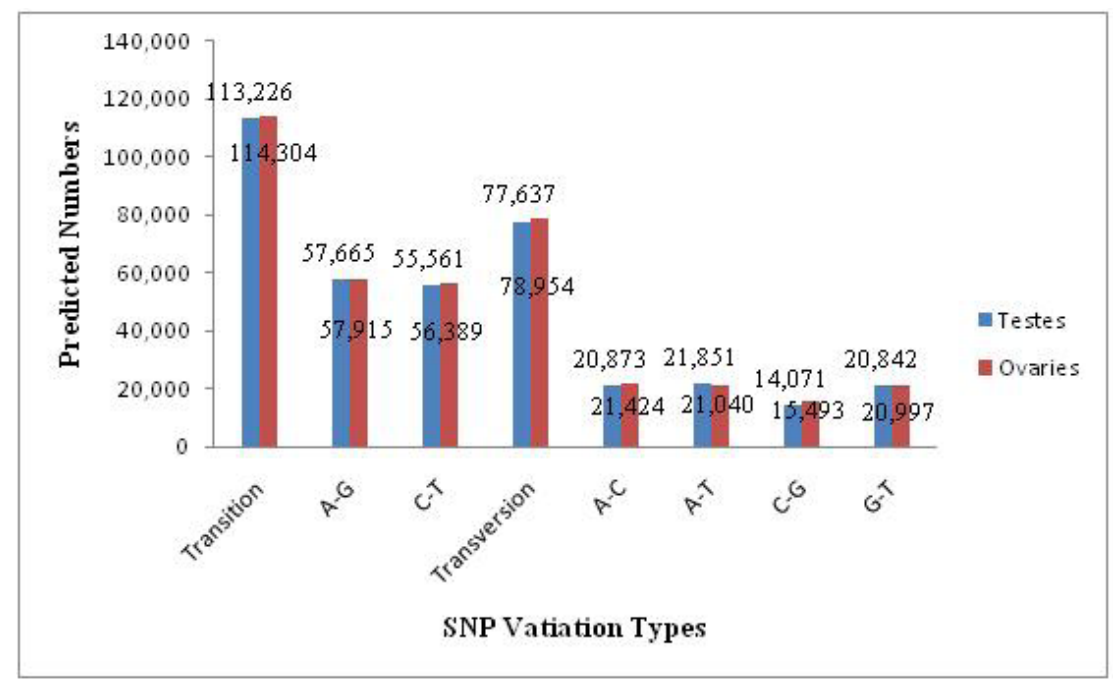

Figure 6. Distribution of putative SNPs in the Acipenser schrenckii transcriptome.

\section{DISCUSSION}

\section{Transcriptome efficiency}

Prior to this study, only 123 cDNAs had been cloned from testicular and ovarian cDNA libraries from A. schrenckii (Chen et al., 2006). A gonad transcriptome for all Acipenser species was constructed for Acipenser fulvescens using Roche 454 GS-FLX. A total of 20,471 unique sequences were obtained with a mean length of $186 \mathrm{bp}$ (Hale et al., 2010). A cDNA library from gonad and brain was constructed for Adriatic sturgeon containing over 55,000 high quality ESTs in order to determine sex-related genes (Vidotto et al., 2013). The lack of a transcriptomic database has delayed studies in A. schrenckii, and even in Acipense species. In the current study, we constructed the first testicular and ovarian transcriptomic database for $A$. Schrenckii using the Illumina HiSeq2000 NGS sequencing platform. The current study generated markedly more genes than was generated by previous studies, taking advantage of the capabilities of Illumina HiSeq2000 NGS, which can achieve more highthroughput sequencing and provide more candidate genes. Furthermore, the average length of each unigene is much longer than that of unigenes identified in previous studies after clustering and de novo assembly. This facilitates further studies on these unigenes, including RT-PCR and western blot analyses, to identify patterns of gene expression. The large numbers of unigenes in this study provide an abundant transcriptome database for use in future analyses of genes related to reproduction and sex determination in A. schrenckii, and may advance such studies conducted on all Acipenser species. Therefore, this transcriptome database accelerates our understanding of the reproductive and sex determination mechanisms in A. schrenckii, and in other Acipenser species.

\section{Annotation analysis}

The total numbers of GO terms and COG categories were much higher than the number of unique sequences, because many contigs can be assigned to more than one GO term or 
category. GO and COG can provide a structured and controlled vocabulary for describing gene products. Reproduction and development-related genes of $A$. schrenckii were most likely to be contained in functional groups in "GO assignments", including "Reproduction", "Developmental process", "Reproductive process", and "Growth", and functional categories in "COG analysis", such as "The general functional prediction only" and "Energy production and conversion". In the current study, genes in these functional groups were significantly more abundant than those in identified in previous studies in Acipenser species (Chen et al., 2006; Hale et al., 2010; Vidotto et al., 2013), providing basic information to aid the further analysis of reproduction and development mechanisms in A. schrenckii and in other Acipenser species.

Furthermore, KEGG analysis dramatically advances research on the relationship between different genes in the transcriptome. A large number of genes involved in reproduction exist in these pathways, including DMRT1, FOXL2, and SOX9. Considering the growth process of $A$. schrenckii, identifying these pathways permits the analysis of reproduction and sex determination mechanisms in A. schrenckii, although not all of the major genes reported in the putative KEGG pathways were found in the current study.

\section{Genes related to reproduction and sex determination}

Research into reproduction and sex determination is needed to maintain the sustainable development for $A$. schrenckii, based on the high economic value of this species. Some homologous genes, which are reported to be involved in reproduction and sex determination in vertebrates, were found from the testicular and ovarian transcriptome.

DMRT1 is required for mammalian testis differentiation, and is reported to be a sexual regulator in worm and fly (Raymond et al., 2000). Sex in birds and chicken is determined by both heterogamous ( $\mathrm{ZW}$ ) and homogamous (ZZ) gemotypes. The $Z$ chromosome is the male-related sex chromosome, whereas the $\mathrm{W}$ chromosome is the female-related sex chromosome. In all birds, previous studies have confirmed that DMRT1 is located on the $Z$ chromosome but is absent from the $\mathrm{W}$ chromosome. In chicken embryos, although DMRT1 is expressed in the early bipotential gonad, it is more highly expressed in males than in females (Raymond et al., 1999). Knockdown of DMRT1 in early chicken embryos can result in the feminization of male gonads (Smith et al., 2009). Recent research showed that DMRT1, which is located on the $Z$ chromosome, plays an essential role in male development in Cynoglossus semilaevis (Chen et al., 2014). A DMRT1 homologous transcript was identified in the transcriptome database in the present study. However, there was no significant difference in its expression between the testes and ovaries. Therefore, the function of this gene in $A$. schrenckii requires further study.

Foxl2 is a member of the forkhead/HNF-3-related family of transcription factors. Its expression is detectable in the mammalian ovaries at the moment of gonadal determination (Crespo et al., 2013). Females lacking the Foxl2 gene appear male. Foxl2 knockout in mature female mice causes them to develop testes, although oocytes are still formed (Uda et al., 2004). Mutations in this gene have been shown to cause blepharophimosis and premature ovarian failure (Ottolenghi et al., 2005). A recent study showed that Foxl2 suppressed testicular differentiation mainly through repression of the SOX9 regulatory element (Uhlenhaut et al., 2009). In vertebrates, Sox9 plays an essential role in the sex determination and developmental process. Sox9 has diverse functions in mammals, including the formation of cartilaginous tissues and testis differentiation. Sox9 promotes the development of male sexual organs through the production of anti-Müllerian hormone (AMH), 
which can inhibit the development of a female reproductive system (De Santa Barbara et al., 1998). Two different Sox9 isoforms, Sox9a and Sox9b, were found in Danio rerio, based on the analysis of differentially expressed genes. Sox9a was only expressed in the testes, whereas Sox9b was only expressed in the ovarian developmental process (Liang et al., 2014). Two different Sox9 isoforms were also found in Oryzias latipes, Salmo irideus, and Pelteobagrus fulvidraco. Both Foxl2 and Sox9 transcripts were found in the transcriptome in the present study. Based on the analysis of differentially expressed genes, Foxl2 was found to be more highly expressed in the ovaries, whereas Sox9 expression was higher in the testes. This implies that these two genes may have similar functions to those found in other vertebrate and mammalian species. The relationship between these two genes needs further research.

FEM1 was first reported to regulate signal-transduction in Caenorhabditis elegans, in which it has diverse functions in the sex determination pathway (Gaudet et al., 1996). The expression of a single FEM1 transcript and protein led to no significant difference in either sex, suggesting that its activity was regulated post-transcriptionally and post-translationally (Gaudet et al., 1996). Its homologs, including FEM1A, FEM1B, and FEM1C, have been found to be involved in sex determination in both human and the house mouse (Mus musculus). In this study, FEM1A, FEM1B, and FEM1C transcripts were identified, suggesting that FEM1 may play an important role in sex determination in $A$. schrenckii.

Vitellogenin is the major precursor of the egg-yolk proteins, which is primarily functioned to form yolk protein, providing nutrients for the developing embryo and larvae (Hook et al., 2007). However, many other biological functions of vitellogenin have also been reported, including temporal division of labor and foraging specialization, regulation of hormonal dynamics and change in gustatory responsiveness (Nelson et al., 2007). Vitellogenin is synthesized in different tissues in different species, and eventually accumulates in the ovary, where it promotes ovarian development (Jeon et al., 2010). Vitellogenin was recently reported to play a role in the host immune system in fish (Garcia et al., 2010). In the present study, vitellogenin and the vitellogenin receptor were not found to be expressed in testes, whereas there was moderate expression in the ovary, suggesting that vitellogenin may be involved in ovarian development. In addition, different isoforms of vitellogenin were found in this study, implying that vitellogenin may have diverse biological functions in $A$. schrenckii and that further research is needed.

Furthermore, many other reproduction-related genes were discovered in the transcriptome. Cathepsin $L$ is involved in yolk processing during vitellogenes is in rainbow trout (Kwon et al., 2001). Cathepsin $C$ was reported to play essential roles in the final stages of oocyte maturation in the kuruma prawn Marsupenaeus japonicus (Qiu et al., 2008). Cathepsin D was found to have high tissue-specific expression in the testis in the rat male reproductive system (Burdan et al., 2006).

\section{Identification of differentially expressed genes involved in reproduction}

Growth hormone $(\mathrm{GH})$ is a peptide hormone that stimulates growth, cell reproduction, and regeneration in humans and other animals. GH promotes growthby increasing the concentration of glucose and free fatty acids (Ranabirand Reetu, 2011). In addition, GH also promotes growth by stimulating the production of insulin-like growth factor 1 (IGF-1) (Scarth, 2006). IGF-1 is generated in target tissues, making it both an endocrine and autocrine hormone (Jansen et al., 1983). In this study, GH was found to have significantly higher expression in ovaries than in testes, implying that $\mathrm{GH}$ has vital functions in promoting growth in females. 
A vasa-like protein transcript was found to be significantly more highly expressed in ovaries than in testes. VASA belong to the DEAD-box family of ATP-dependent RNA helicases, which have diverse functions in germ cell development (Lasko and Ashburner, 1988). The product of this gene is required maternally for germplasm assembly and completion of oogenesis. VASA is considered a reliable molecular marker that can be used to trace the origin, migration, and differentiation of primordial germ cells because it is specifically and stably expressed in germ cells in most animals (Chang et al., 2002). Higher expression in the ovariessuggests that the vasa-like protein may also have an important role in the promotion of germplasm development and in the completion of oogenesis in A. schrenckii.

Cytochrome P450 (CYP) belongs to a superfamily of proteins that contain a hemecofactor. CYP enzymes have been identified in alliving organisms (Lamb et al., 2009) except for E. coli (Sigel et al., 2007). Most CYP enzymes require a protein partner to deliver one or more electrons to reduce iron and eventually oxygen. The expression levels of different types of CYPs varied in the testes and ovaries of $A$. schrenckii. For example, Cytochrome P450 is more highly expressed in testes, whereas cytochrome $\mathrm{P} 4501 \mathrm{~B}$ is more highly expressed in ovaries, implying that members of the CYP family have diverse functions in A. schrenckii.

\section{Identification of SSR and SNP markers}

SSRs, or microsatellites, are polymorphic loci present in genomic DNA that consist of repeated core sequences of 2-6 base pairs in length (Queller et al., 1993). SNPs represent the most common type of variation in the genome. SNPs provide the best genome coverage for analyzing the performance and production of traits. Genomes with high-density SNP coverage are powerful tools for whole-genome association studies because they permit the detection of linkage disequilibrium (Wang et al., 1998). Thus, SSR and SNP markers have been widely used in molecular genetic research, such as evolution, molecular ecology, and quantitative trait loci (QTL) analysis.

Considering the advantages of SSRs and SNPs, the development of such markers to analyze the evolution, molecular ecology, and QTLs from A. schrenckii is desirable. The potential markers identified in this study will provide an invaluable resource for such studies. SSR and SNP markers will be identified from the transcriptomic database in future studies.

In conclusion, a comparative testicular and ovarian transcriptome of $A$. schrenckii was constructed in this study. This provides new sequence information for $A$. schrenckii, which will provide a basis for further genetic studies of Acipenser species. Many genes related to reproduction and development were identified in this study, which improves our understanding of reproductive and developmental mechanisms in A. schrenckii and Acipenser species. Potential SSR and SNP markers isolated from the transcriptome may shed light on the evolution and molecular ecology of Acipenser species. These data will advance research in the field of Acipenser molecular genetics.

\section{Data deposition}

The IlluminaHiSeq2000 reads of $A$. schrenckii were submitted to http://www.hrfri.ac.cn/ news_nei.asp?newsid=1508\&SortID=12\&SortPath=0,12.

\section{Conflicts of interest}

The authors declare no conflict of interest. 


\section{ACKNOWLEDGMENTS}

We thank International Science Editing for their help revising the English. Research supported by the National Natural Science Foundation of China (Grant \#31201971) and the National Science \& Technology Supporting Program of the 12th Five-year Plan of China (Grant \#2012BAD25B10).

\section{REFERENCES}

Burdan F, Szumiło J, Dudka J, Dąbrowski A, et al. (2006). The activity and immunoexpression of cathepsin D in rat male reproductive organs. Folia Morphol. 65: 111-115.

Chang CC, Dearden P and Akam M (2002). Germ line development in the grasshopper Schistocerca gregaria: vasa as a marker. Dev. Biol. 252: 100-118.

Chen J, Wang B, Wu W, Liang B, et al. (2006). Cloning and screening of differentially expressed genes from testis and ovariesof Amur sturgeon. J. Fish. Sci. China 13: 8-12.

Chen SL, Zhang GJ, Shao CW, Huang QF, et al. (2014). Whole-genome sequence of a flatfish provides insights into ZW sex chromosome evolution and adaptation to a benthic lifestyle. Nat. Genet. 46: 253-260.

Cloonan N and Grimmond SM (2008). Transcriptome content and dynamics at single nucleotide resolution. Genome Biol. 9: 234.

Crespo B, Lan-Chow-Wing O, Rocha A, Zanuy S, et al. (2013). foxl2 and foxl3 are two ancient paralogs that remain fully functional in teleosts. Gen. Comp. Endocrinol. 19: 481-93.

De Santa Barbara P, Bonneaud N, Boizet B, Desclozeaux M, et al. (1998). Direct interaction of SRY-related protein SOX9 and steroidogenic factor 1 regulates transcription of the human anti-Müllerian hormone gene. Mol. Cell Biol. 18: 6653-6665.

Garcia J, Munro ES, Monte MM, Fourrier MC, et al. (2010). Atlantic salmon (Salmo salar L.) serum vitellogenin neutralises infectivity of infectious pancreatic necrosis virus (IPNV). Fish Shellfish Immunol. 29: 293-297.

Gaudet J, VanderElst I and Spence AM (1996). Post-transcriptional regulation of sex determination in Caenorhabditis elegans: widespread expression of the sex determining gene fem-1 in both sexes. Mol. Biol. Cell 7: 1107-1121.

Hale MC, Jackson JR and DeWoody JA (2010). Discovery and evaluation of candidate sex-determining genes and xenobiotics in the gonads of lake sturgeon (Acipenser fulvescens). Genetica 138: 745-756.

Hook SE, Skillman AD, Small JA and Schultz IR (2007). Temporal changes in geneexpression in rainbow trout exposed to ethynyl estradiol. Comp. Biochem. Physiol. C Toxicol. Pharmacol. 145: 73-85.

Huang W and Marth G (2008). A genome assembly viewer for next-generation sequencing technologies. Genome Res. 18: 1538-1543.

Jansen M, van Schaik F, Ricker A, Bullock B, et al. (1983). Sequence of cDNA encoding human insulin-like growth factor I precursor. Nature 306: 609-611.

Jeon JM, Lee SO, Kim KS, Baek HJ, et al. (2010). Characterization of two vitellogenin cDNAs from a Pandalus shrimp (Pandalopsis japonica): expression in hepatopancreas is down-regulated by endosulfan exposure. Comp. Biochem. Physiol. B Biochem. Mol. Biol. 157: 102-112.

Kwon JY, Prat F, Randall C and Tyler CR (2001). Molecular characterization of putative yolk processing enzymes and their expression during oogenesis and embryogenesis in rainbow trout (Oncorhynchus mykiss). Biol. Reprod. 65: 1701-1709.

Lamb DC, Lei L, Warrilow AG, Lepesheva GI, et al. (2009). The first virally encoded cytochrome P450. J. Virol. 83: 8266-8269.

Lasko PF and Ashburner M (1988). The product of the Drosophila gene vasa is very similar to eukaryotic initiation factor-4A. Nature 335: 611-617.

Li D (2011). RSEM: accurate transcript quantification from RNA-Seq data with or without a reference genome. BMC Bioinformatics 12: 323.

Li D, Liu Z and Xie C (2012). Effect of stocking density on growth and serum concentrations of thyroid hormones and cortisol in Amur sturgeon, Acipenser schrenckii. Fish Physiol. Biochem. 38: 511-520.

Liang QY, Wang XY, Sun DJ and Yan JZ (2014). Dynamic expression patterns of two Sox9 genes in developing embryo and gonads of zebra fish. Shandong Agric. Sci. 46: 20-24.

Ludwig A (2008). Identification of Acipenseri formes species in trade. J. Appl. Ichthyol. 24: 2-19.

Mardis E (2008). The impact of next-generation sequencing technology on genetics. Trends Genet. 24: 133-141.

Morozova O and Marra MA (2008). Applications of next-generation sequencing technologies in functional genomics. Genomics 92: $255-264$ 
Nelson CM, Ihle KE, Fondrk MK, Page RE, et al. (2007). The gene vitellogenin has multiple coordinating effects on social organization. PLoS Biol. 5: e62.

Ottolenghi C, Omari S, Garcia-Ortiz JE, Uda M, et al. (2005). Foxl2 is required for commitment to ovariesdifferentiation. Hum. Mol. Genet. 14: 2053-2062.

Qiu LH, Jiang SG, Huang JH, Wang W, et al. (2008). Molecular cloning and mRNA expression of cathepsin C gene in black tiger shrimp (Penaeus monodon). Comp. Biochem. Physiol. A Mol. Integr. Physiol. 150: 320-325.

Queller DC, Strassman JE and Hughes CR (1993). Microsatellites and kinship. Trends Ecol. Evol. 8: 285-288.

Ranabir S and Reetu K (2011). Stress and hormones. Indian J. Endocrinol. Metab. 15: 18-22.

Raymond CS, Kettlewell JR, Hirsch B, Bardwell VJ, et al. (1999). Expression of Dmrt1 in the genital ridge of mouse and chicken embryos suggests a role in vertebrate sexual development. Dev. Biol. 215: 208-220.

Raymond CS, Murphy MW, O'Sullivan MG, Bardwell VJ, et al. (2000). Dmrt1, a gene related to worm and fly sexual regulators, is required for mammalian testis differentiation. Genes Dev. 14: 2587-2595.

Sigel R, Sigel A and Sigel H (2007). The ubiquitous uolesof cytochrome P450 proteins: metal ions in life sciences. Wiley, New York.

Scarth J (2006). Modulation of the growth hormone-insulin-like growth factor (GH-IGF) axis by pharmaceutical, nutraceutical and environmental xenobiotics: an emerging role for xenobiotic-metabolizing enzymes and the transcription factors regulating their expression. A review. Xenobiotica 36: 119-218.

Smith CA, Roeszler KN, Ohnesorg T, Cummins DM, et al. (2009). The avian Z-linked gene DMRT1 is required for male sex determination in the chicken. Nature 461: 267-271.

Uda M, Ottolenghi C, Crisponi L, Garcia JE, et al. (2004). Foxl2 disruption causes mouse ovarian failure by pervasive blockage of follicle development. Hum. Mol. Genet. 13: 1171-1181.

Uhlenhaut NH, Jakob S, Anlag K, Eisenberger T, et al. (2009). Somatic sex reprogramming of adult ovaries to testes by FOXL2 ablation. Cell 139: 1130-1142.

Vidotto M, Grapputo A, Boscari E, Barbisan F, et al. (2013). Transcriptome sequencing and de novo annotation of the critically endangered Adriatic sturgeon. BMC Genomics 14: 407.

Wang DG, Fan JB, Siao CJ, Berno A, et al. (1998). Large-scale identification, mapping, and genotyping of single-nucleotide polymorphisms in the human genome. Science 280: 1077-1082.

Wang D, Lu TY and Liu HB (2009a). Characterization and genetic diversity of the sturgeon Acipenser schrenckii. Ig heavy chain. Immunobiology 214: 359-366.

Wang Z, Gerstein M and Snyder M (2009b). RNA-Seq: a revolutionary tool for transcriptomics. Nat. Rev. Genet. 10: 57-63.

Wei QW, Zou Y, Li P and Li L (2011). Sturgeon aquaculture in China: progress, strategies and prospects assessed on the basis of nation-wide surveys (2007-2009). J. Appl. Ichthyol. 27: 162-168. 